\title{
Electric Charge, Matter Distribution, and Baryon Asymmetry from Holographic Principle
}

\author{
T. R. Mongan \\ 84 Marin Avenue, Sausalito, CA, USA \\ Email: tmongan@gmail.com
}

How to cite this paper: Mongan, T.R. (2021) Electric Charge, Matter Distribution, and Baryon Asymmetry from Holographic Principle. Journal of Modern Physics, 12, 295-299.

https://doi.org/10.4236/jmp.2021.123022

Received: January 26, 2021

Accepted: February 23, 2021

Published: February 26, 2021

Copyright $\odot 2021$ by author(s) and Scientific Research Publishing Inc. This work is licensed under the Creative Commons Attribution International License (CC BY 4.0).

http://creativecommons.org/licenses/by/4.0/

\begin{abstract}
Explaining baryon asymmetry (i.e., matter dominance) in the universe has been a vexing problem in physics. This analysis, based on the holographic principle, identifies fractional electric charge with the state of bits of information on the event horizon. Thermodynamics on the event horizon at the time of baryogenesis then estimates observed baryon asymmetry.
\end{abstract}

\section{Keywords}

Electric Charge, Baryon Asymmetry, Holographic Principle

\section{Introduction}

Baryon asymmetry (matter dominance) in the universe has been difficult to explain. This paper is based on the holographic principle [1], a direct consequence of quantum mechanics, general relativity, black hole thermodynamics, and Shannon information theory, indicating only a finite number (about $10^{122}$ ) of bits of information encoded on the event horizon will ever be available to describe our observable universe. The two possible states of each bit, positive and negative, can be identified with fractional charge in the universe. Describing Standard Model fermions as spheres with radius 1/4 their Compton wavelength [2] indicates charge $\pm \frac{e}{6}$ associated with each bit, where $e$ is electron charge. Thermodynamics on the event horizon at the time of baryogenesis and energy difference between bit states then estimates observed baryon asymmetry. Similar treatment of baryon asymmetry [3] used a specific preon model not relevant to this analysis. 


\section{Charge and the Holographic Principle}

Fundamental particles in the Standard Model have charge $\pm \frac{n e}{3}$, where $n=0,1$, 2 or 3. Considering fundamental fermions as spheres with radius 1/4 their Compton wavelength [2] requires surface charge $\pm \frac{n e}{6}$ at each end of the spin axis to insure identical electrostatic potential energy of first-generation fermions. So, identifying states of each bit of information available to describe the observable universe as $\frac{e}{6}$ or $-\frac{e}{6}$ accounts for charge in the universe. Origin of the universe by quantum fluctuation from nothing [4] requires equal number of plus and minus charged bits to ensure a charge-neutral universe and charge conservation, a precondition for gauge invariance and Maxwell's equations. In any physical system, energy must be transferred to change state of information bits. Protons have charge $e$ and anti-protons have charge $-e$. Regardless of how bits of information on the horizon specify protons or anti-protons, bit configurations specifying protons must differ in 6 bits from configurations specifying anti-protons. Since $\frac{e}{6}$ bits and $-\frac{e}{6}$ bits do not have the same energy, the number of protons and anti-protons created in the early universe must be slightly different. If $\frac{e}{6}$ bits have lower energy than $-\frac{e}{6}$ bits, there must be more matter than anti-matter in the universe and this analysis estimates that baryon asymmetry.

\section{Matter Distribution in the Universe}

At the fundamental level, information specifies time evolution of matter distribution in the universe. Observations indicate our universe is a closed Friedmann universe, dominated by vacuum energy in the form of a cosmological constant and so large it is approximately flat [5]. If Hubble constant $H_{0}=67.8 \mathrm{~km} \cdot \mathrm{sec}^{-1} \cdot \mathrm{Mpc}^{-1}$, critical density $\rho_{\text {crit }}=\frac{3 H_{0}^{2}}{8 \pi G}=8.64 \times 10^{-30} \mathrm{~g} / \mathrm{cm}^{3}$, where $G=6.67 \times 10^{-8} \mathrm{~cm}^{3} /\left(\mathrm{g} \cdot \mathrm{sec}^{2}\right)$. Matter accounts for $30.8 \%$ of energy in today's universe, today's matter density in the universe is $\rho_{0}(0)=0.308 \rho_{\text {crit }}=2.66 \times 10^{-30} \mathrm{~g} / \mathrm{cm}^{3}$, and vacuum energy density $\rho_{v}=(1-0.308) \rho_{\text {crit }}=5.98 \times 10^{-30} \mathrm{~g} / \mathrm{cm}^{3}$. Cosmological constant $\Lambda=\frac{8 \pi G \rho_{v}}{c^{2}}$, where $\mathrm{c}=3 \times 10^{10} \mathrm{~cm} / \mathrm{sec}$, and there is an event horizon in the universe at radius $R_{H}=\sqrt{\frac{3}{\Lambda}}=1.64 \times 10^{28} \mathrm{~cm}$. The holographic principle then indicates only $N=\frac{\pi R_{H}^{2}}{\ln (2) l_{P}^{2}}=4.69 \times 10^{122}$ bits of information on the event horizon will ever be able to describe our observable universe, where Planck length $l_{P}=\sqrt{\frac{\hbar G}{c^{3}}}=1.62$ $\times 10^{-33} \mathrm{~cm}$ and Planck's constant $\hbar=1.05 \times 10^{-27} \mathrm{~g} \cdot \mathrm{cm}^{2} / \mathrm{sec}$.

With no source or sink for information outside a closed universe, the amount 
of information available to describe the observable universe remains constant. In a closed universe, the total quantity of matter in the universe is conserved, there are only $N$ bits of information available and mass per bit of information, approximately $10^{-67} \mathrm{~g}$, is mass $M_{H}=\frac{4}{3} \pi R_{H}^{3} \rho_{0}(0)=4.92 \times 10^{55} \mathrm{~g}$ divided by the number of bits $N$.

Information on the horizon specifying distribution of mass within the observable universe at any instant is of little use to an observer, because signals traveling at light speed take about 17 billion years to reach the observer. However, as shown below, baryon asymmetry can be estimated using only information on the horizon at the time of baryogenesis. Since mass associated with each bit of information is only about $10^{-67} \mathrm{~g}$, the analysis is consistent with protons and anti-protons having indistinguishable masses.

\section{Baryon Asymmetry}

Temperature at time of baryon formation (baryogenesis) was $T_{B}=\frac{2 m_{p} c^{2}}{k}=2.18 \times 10^{13}{ }^{\circ} \mathrm{K}$, where Boltzmann constant $k=1.38 \times 10^{-16}\left(\frac{\mathrm{g} \cdot \mathrm{cm}^{2}}{\mathrm{sec}^{2}}\right) /{ }^{\circ} \mathrm{K}$ and proton mass $m_{p}=1.67 \times 10^{-24} \mathrm{~g}$. Scale factor of the universe at baryogenesis was $R_{B}=R_{0}\left(\frac{2.725}{T_{B}}\right) \approx 10^{15} \mathrm{~cm}$, where $2.725{ }^{\circ} \mathrm{K}$ is today's cosmic microwave background temperature and $R_{0} \approx 10^{28} \mathrm{~cm}$ is today's scale factor. Time $t_{B}$ of baryogenesis, in seconds after the end of inflation, is found from Friedmann's equation $\left(\frac{\mathrm{d} R}{\mathrm{~d} t}\right)^{2}-\left(\frac{8 \pi G}{3}\right) \varepsilon\left(\frac{R}{c}\right)^{2}=-\kappa c^{2}$. After inflation, the universe is so large it is almost flat, and curvature parameter $\kappa \approx 0$. Energy density is $\varepsilon(R)=\varepsilon_{r}\left(\frac{R_{0}}{R}\right)^{4}+\varepsilon_{m}\left(\frac{R_{0}}{R}\right)^{3}+\varepsilon_{v}$, where $\varepsilon_{r}=4.4 \times 10^{-34}\left(\frac{\mathrm{g}}{\mathrm{cm}^{3}}\right) c^{2}, \varepsilon_{m}$, and $\varepsilon_{v}$ are today's radiation, matter, and vacuum energy densities. Vacuum energy density was negligible in the early post-inflationary universe, and radiation dominated before radiation/matter equality, when $R \ll 10^{-5} R_{0}$. Integrating $\left(\frac{\mathrm{d} R}{\mathrm{~d} t}\right)^{2}-\left(\frac{8 \pi G}{3 c^{2}}\right) \frac{\varepsilon_{r} R_{0}^{4}}{R^{2}}=\left(\frac{\mathrm{d} R}{\mathrm{~d} t}\right)^{2}-\left(\frac{A}{R}\right)^{2}=0$, where $A=\sqrt{\frac{8 \pi G \varepsilon_{r} R_{0}^{4}}{3 c^{2}}}$, from the end of inflation at $t=0$ to $t$ gives $\frac{1}{2}\left(R^{2}-R_{i}^{2}\right)=A t$, where $R_{i}$ is scale factor at the end of inflation. So $t_{B}=\frac{R_{B}^{2}-R_{i}^{2}}{2 A} \approx \frac{R_{B}^{2}}{2 A} \approx 10^{-7} \mathrm{sec}$, if $R_{B} \gg R_{i}$. Distance from any point in the universe to the particle horizon for that point [6] is $d_{B}=c R_{B} \int_{0}^{t_{B}} \frac{d t^{\prime}}{R\left(t^{\prime}\right)}=\left[\frac{c R_{B}}{A} \sqrt{R_{i}^{2}+2 A t}\right]_{0}^{t_{B}}=\frac{c R_{B}}{A}\left[\sqrt{R_{i}^{2}+2 A t_{B}}-R_{i}\right]$. Since $R_{B} \gg R_{i}$,
$d_{B} \approx c R_{B} \sqrt{\frac{2 t_{B}}{A}} \approx 10^{4} \mathrm{~cm}$. 
Surface gravity on particle horizon at baryogenesis is

$$
g_{H B}=\frac{4 \pi G}{3 c^{2}} \varepsilon\left(R_{B}\right) d_{B} \approx \frac{4 \pi G}{3 c} \frac{\varepsilon_{r} R_{0}^{4}}{A R_{B}^{2}}
$$

and associated horizon temperature [7] [8] is

$$
T_{H B}=\frac{\hbar}{2 \pi c k} g_{H B} \approx 6 \times 10^{-7} \mathrm{~K}
$$

Temperature at any epoch is uniform throughout a postinflationary homogeneous isotropic Friedman universe, and causal horizon at baryogenesis is distance $d_{B}$ from every point in the universe. Temperature at every point on the causal horizon for every point in the universe is the same because surface gravity of the uniform sphere within the horizon is the same at every point on every horizon. Bits on all causal horizons are in thermal equilibrium, only two quantum states are accessible to those bits, and equilibrium statistical mechanics establishes occupation probabilities of bit states in thermal equilibrium at temperature $T_{H B}$ proportional to their corresponding Boltzmann factors. So, if energy of $\frac{e}{6}$ bits on the horizon at baryogenesis is $E_{b i t}-E_{d}$ and energy of $-\frac{e}{6}$ bits is $E_{b i t}+E_{d}$, proton/antiproton ratio at baryogenesis is $\left(e^{-\frac{E_{b i t}-E_{d}}{k T_{H B}}} / e^{-\frac{E_{b i t}+E_{d}}{k T_{H B}}}\right)^{6}=e^{\frac{12 E_{d}}{k T_{H B}}} \approx 1+\frac{12 E_{d}}{k T_{H B}}$ and proton excess is $\frac{12 E_{d}}{k T_{H B}}$.

Energy released when a bit on the horizon drops from $-\frac{e}{6}$ state to $\frac{e}{6}$ state raises another bit from $\frac{e}{6}$ to $-\frac{e}{6}$ state, and that is the mechanism for charge conservation. Energy must be transferred by massless quanta with wavelengths related to the size of the universe. With no reliable definition of size (as opposed to scale factor) of an open universe, this analysis only applies to a closed Friedmann universe, in particular one like ours that is so large it is approximately flat. The only macroscopic length characteristic of the horizon of a closed Friedmann universe with radius (scale factor) $R(t)$ is circumference $2 \pi R(t)$. If energy $2 E_{d}$ to change the state of bits associated with a mass quantum within the universe (and corresponding bits on the horizon) at baryogenesis equals the energy of massless quanta with wavelength characteristic of a closed Friedmann universe with radius $R_{B}$

$$
2 E_{d}=\frac{\hbar c}{R_{B}}
$$

Using Equations (1), (2), and (3), proton excess at baryogenesis is $\frac{12 E_{d}}{k T_{H B}}=\left(\frac{24 \pi c^{2}}{R_{0}}\right)\left(\frac{2.725}{T_{B}}\right) \sqrt{\frac{3}{8 \pi G \varepsilon_{r}}}$. Dependence on $R_{0}$ arises because $R_{B}$, the radius of the universe at baryogenesis, depends on $R_{0}$, today's cosmic microwave background temperature $2.725^{\circ} \mathrm{K}$, and temperature $T_{B}$ at baryogenesis. For $R_{0} \approx$ $10^{28} \mathrm{~cm}$, proton excess is $1.8 \times 10^{-9}$. WMAP [9] estimated baryon density to cosmic 
microwave background photon density ratio as $6.1 \times 10^{-10}$. At baryogenesis, the number of proton states with six $\frac{e}{6}$ bits approximately equals the number of anti-protons states with six $-\frac{e}{6}$ bits, and when almost all protons and antiprotons annihilate to two photons the ratio of baryon to photon states is $\frac{1}{3}\left(1.8 \times 10^{-9}\right)=6 \times 10^{-10}$, in agreement with WMAP.

\section{Conclusion}

Using the holographic principle, states of information bits on the event horizon describing matter distribution within the universe are identified with fractional electric charge. Describing fundamental fermions in the Standard Model as spheres with radius $1 / 4$ their Compton wavelength and half their charge on the surface at each end of their spin axis, combined with thermodynamics on the event horizon at the time of baryogenesis, explains baryon asymmetry.

\section{Acknowledgements}

This note was inspired by discussions with the young scientist James Bartley Mongan.

\section{Conflicts of Interest}

The author declares no conflicts of interest regarding the publication of this paper.

\section{References}

[1] Bousso, R. (2002) Reviews of Modern Physics, 74, 825. https://doi.org/10.1103/RevModPhys.74.825

[2] Mongan, T.R. (2020) Journal of Modern Physics, 11, 1993-1998. https://doi.org/10.4236/jmp.2020.1112126

[3] Mongan, T.R. (2012) Journal of Modern Physics, 3, 581-584. https://doi.org/10.4236/jmp.2012.37079

[4] Mongan, T.R. (2001) General Relativity and Gravitation, 33, 1415-1424. https://doi.org/10.1023/A:1012065826750

[5] Mongan, T.R. (2015) Holography, Large Scale Structure, Supermassive Black Holes and Minimum Stellar Mass. arXiv: 1301.0304.

[6] Islam, J. (2002) An Introduction to Mathematical Cosmology. 2nd Edition, Cambridge University Press, Cambridge, p. 73.

[7] Padmanabhan, T. (2010) AIP Conf. Proc., 1241, 93. [arXiv:0911.1403].

[8] Padmanabhan, T. (2009) A Dialogue on the Nature of Gravity. [arXiv: 0910.0839].

[9] Bennet, C.L., et al. (2003) The Astrophysical Journal Supplement Series, 148, 1. 